\title{
Staining of Eosinophils with Nitroblue Tetrazolium in Patients with Chronic Granulomatous Disease
}

\author{
ROBERT L. ROBERTS, YOHICHIROH OHNO, AND JOHN I. GALLIN \\ Bacterial Diseases Section, Laboratory of Clinical Investigation, National Institute of Allergy and Infectious \\ Diseases, National Institutes of Health, Bethesda, Maryland 20892
}

\begin{abstract}
The nitroblue tetrazolium (NBT) test is the primary screening test for chronic granulomatous disease (CGD). Neutrophils and other phagocytic cells readily reduce NBT to blue formazan after oxidative stimulation whereas CGD cells remain colorless. In the present study purified eosinophil populations were obtained from CGD patients and normals by exposing peripheral blood to the peptide $\mathrm{N}$-formyl-methionyl-leucyl-phenylalanine and then centrifuged over a discontinuous Percoll gradient. The eosinophils were then incubated in $0.1 \%$ NBT $\left(37^{\circ} \mathrm{C}, 15\right.$ min) with either phorbol myristate acetate or buffer alone (HEPES with calcium and magnesium). Blue staining characteristic of NBT reduction occurred in the phorbol myristate acetate-stimulated eosinophils of half $(4 / 8)$ of the CGD patients tested. The staining was most intense between the nuclear lobes of the eosinophil with little staining near the cell periphery. The staining pattern was present in $75.5 \pm 5.3 \%$ of the purified eosinophils in those patients in which the phenomenon occurred and was reproducible in the same patients over a 6-month period. Eosinophils from normal individuals tested with NBT and phorbol myristate acetate showed intense staining over the entire cell cytoplasm as did normal neutrophils. Purified CGD eosinophils that did show the staining pattern were not able to produce superoxide (as measured by cytochrome $\mathrm{C}$ reduction) when stimulated by phorbol myristate acetate indicating the staining was probably not related to superoxide production. Patients with CGD have a mild eosinophilia (4.6 $\pm 0.7 \%$ in 11 patients at the National Institutes of Health) and eosinophil staining may account for the small number of positive NBT cells reported in some patients. (Pediatr Res 20: 378-380, 1986)
\end{abstract}

\section{Abbreviations}

CGD, chronic granulomatous disease

NBT, nitroblue tetrazolum

PMA, phorbol myristate acetate

FMLP, N-formyl-methionyl-leucyl-phenylalanine

Patients with CGD are highly susceptible to catalase-positive microorganisms due to the inability of their neutrophils to generate superoxide and $\mathrm{H}_{2} \mathrm{O}_{2}$. Defects or the absence of one of the various enzymes required to generate superoxide has been shown to be the basis of this disease which may be inherited in an Xlinked or autosomal pattern (1). The reduction of NBT is often

Received July 1, 1985; accepted December 11, 1985

Correspondence Dr. Robert L. Roberts, Department of Pediatrics, Marion Davies Children Center, UCLA CHS, Los Angeles, CA 90024. the primary screening test for CGD. Neutrophils from normal individuals readily reduce NBT to blue formazan after exposure to zymosan or a soluble stimulus such as PMA. In contrast CGD neutrophils will remain colorless, indicating inability to supply the oxygen radicals needed to reduce the dye although some patients have been reported to have a low percentage of NBT positive granulocytes (2).

In the present study purified eosinophil and unfractionated granulocyte populations from CGD patients were tested for PMA-stimulated NBT reduction. The neutrophils remained colorless as expected, but eosinophils from half of the patients showed blue staining characteristic of NBT reduction.

\section{METHODS}

Patients were referred to the National Institutes of Health for recurrent infections and ranged in age from 3 to $25 \mathrm{yr}$. The diagnosis of CGD was confirmed by NBT testing, impaired killing of Staphylococcus aureus by the patient's granulocytes, and inability to generate superoxide by cytochrome $c$ reduction. For most studies patients were free of infection at the time of study. Infected patients were also studied and there were no differences in the results. Blood from four patients with parasitosis (microfilariasis) was kindly provided by Drs. Eric Ottesen and Thomas Nutman.

Purified eosinophils were obtained from patients and adult volunteers by exposing peripheral blood to the chemotactic peptide FMLP which stimulates and decreases the density of the neutrophils but does not alter the eosinophils (3). The blood was then centrifuged over a discontinuous Percoll gradient yielding greater than $90 \%$ eosinophils between the $65 \%$ and $75 \%$ layers of Percoll. Granulocytes were obtained by discontinuous Percoll gradient centrifugation without exposure to FMLP. NBT testing with PMA $(20 \mu \mathrm{g} / \mathrm{ml})$ was then performed as previously described (4) and at least 300 cells were counted to determine the percentage NBT positive. Superoxide production was determined by superoxide dismutase inhibitable cytochrome $c$ reduction (5). Cytochrome $b$ was measured from difference spectra of PMN sonicates treated with azide plus hydrogen peroxide as recently described (6). FAD was determined in particulate fractions from activated neutrophils as previously described (7) and the method of Faeder and Siegel (8) to quantitate FAD.

\section{RESULTS}

Granulocytes from 11 normal individuals, which were tested simultaneously with the patients, were consistently greater than 95\% positive for NBT staining. Purified eosinophils from normals as well as patients with parasitosis were also greater than 95\% positive after PMA stimulation. Unfractionated granulocytes and purified eosinophils were less than $5 \%$ positive for NBT staining in the absence of PMA. 
The results of NBT testing after PMA stimulation for the unfractionated granulocytes and purified eosinophils from CGD patients is shown in Table 1. In half of the patients no staining of either granulocytes or purified eosinophils was observed. Eosinophils from the other half of the patients did show blue staining characteristic of NBT reduction in $75.5 \pm 5.3 \%$ of their cells. In the same patients staining was also noted in the granulocyte fraction but to a much lesser degree. As can be seen four of the eight patients studied had X-linked CGD; each of these patients' neutrophils lacked cytochrome $b$ in contrast of the patients with autosomal inheritance who had normal amounts of cytochrome b. FAD was present in each patients' neutrophils. In two patients, MB and JH, FAD measurements were performed on subcellular fractions including cytosolic, plasma membrane, specific granule enriched and azurophilic granule enriched fractions and no deficiency was noted (data not shown). Furthermore, there was no correlation between the pattern of inheritance, neutrophil cytochrome b, or FAD content, and the extent of NBT reduction by the patient's eosinophils.

The pattern of staining in CGD eosinophils was different from that of normal eosinophils (Fig. 1). In the normal eosinophils (A) patches of reduced NBT are scattered throughout the cytoplasm, extending to the periphery. This is the same type staining found in normal neutrophils. In the CGD eosinophils $(B)$ the staining was most intense between the nuclear lobes with no staining near the periphery. The staining did not occur in CGD

Table 1. NBT staining of unfractionated granulocytes and purified eosinophils in CGD patients

\begin{tabular}{|c|c|c|c|c|c|c|c|}
\hline Patient & Sex & Date & Inheritance* & $\begin{array}{c}\text { Cytochrome b } \\
\left(\mathrm{pmol} / 7.5 \times 10^{6}\right. \\
\text { cells }) \dagger\end{array}$ & $\begin{array}{c}\text { FAD } \\
(\mathrm{pmol} / \\
\text { mg } \\
\text { protein }) \ddagger\end{array}$ & \multicolumn{2}{|c|}{ Stained cells $(\%) \S$} \\
\hline RW & & $3 / 21 / 84$ & & & & 6 & 74 \\
\hline DHo & M & $11 / 17 / 83$ & $X L$ & 0 & 120 & 0 & 0 \\
\hline $\mathrm{JHe}$ & M & $11 / 23 / 83$ & Auto & 20 & 48 & 3 & 65 \\
\hline $\mathrm{BJ}$ & $M$ & $2 / 28 / 84$ & Auto & 32 & 153 & 0 & 0 \\
\hline RK\| & M & $3 / 5 / 84$ & $X L$ & 0 & 55 & 0 & 0 \\
\hline PK\| & M & $3 / 5 / 84$ & $X L$ & 0 & 68 & 0 & 0 \\
\hline \multirow[t]{2}{*}{$\mathrm{MB}$} & M & $3 / 12 / 84$ & Auto & 40 & 54 & 1 & 82 \\
\hline & & $5 / 30 / 84$ & & & & 2 & 67 \\
\hline
\end{tabular}

* Refers to sex-linked (XL) or autosomal (Auto) pattern of inheritance.

$\dagger$ Cytochrome b measured as described in the methods; the mean cytochrome b in neutrophils from 24 normal subjects was $47 \pm 3$ pmol/7.5 $\times$ $10^{6}$ cells.

$\ddagger F A D$ in particulate fractions of neutrophils was measured as described in the "Methods"; the mean FAD in neutrophils from 11 normal subjects was $105 \pm 9 \mathrm{pmol} / \mathrm{mg}$ protein. Only the value for patient RW is significantly outside the normal values $(p<0.05)$.

$\S$ Based on count of at least 300 cells.

\| Brothers.
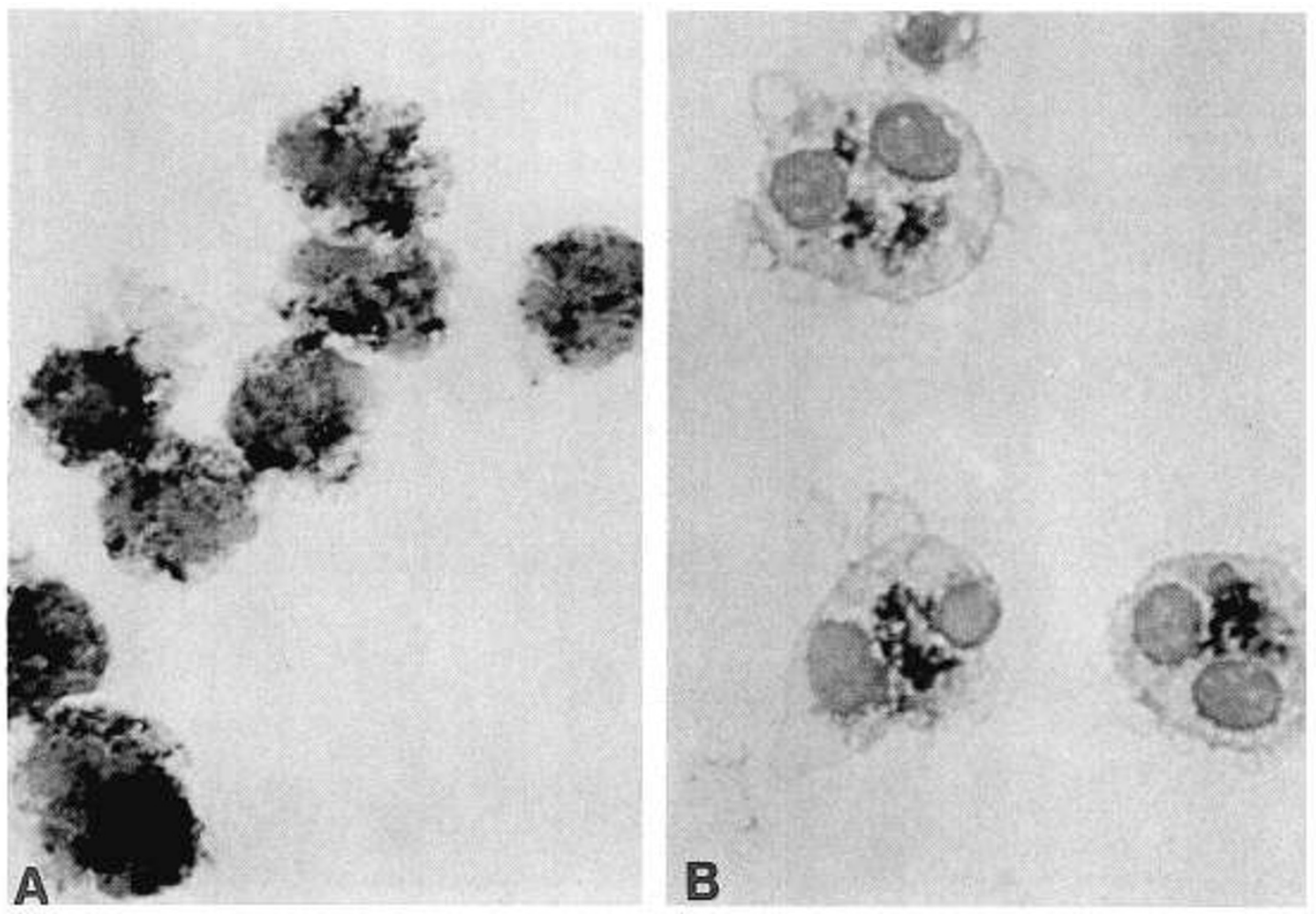

Fig. 1. Eosinophils from normal adult $(A)$ and CGD patient $(B)$ after exposure to NBT and PMA $(20 \mathrm{ng} / \mathrm{ml})$ for $15 \mathrm{~min}$ at $37^{\circ} \mathrm{C}$. The cells were counterstained with safranine. 
eosinophils exposed to only $0.1 \%$ DMSO, the solvent for PMA. Exposure to PMA altered the typical bilobed nuclear morphology of eosinophils, similar to the effect of PMA on neutrophil morphology (9) although the normal eosinophils appeared more greatly affected (Fig. 1A).

Counterstaining unfractionated granulocytes from CGD patients with Wright's stain after NBT and PMA exposure confirmed that staining occurred only in eosinophils and that all CGD neutrophils were negative. This indicates that exposure to FMLP during eosinophil purification was not necessary for the staining to occur as the unfractionated cells had not been treated with FMLP.

Eosinophils from one patient whose eosinophils did reduce NBT (MB) were isolated and their ability to produce superoxide, as measured by cytochrome $c\left(7.0 \times 10^{-5} \mathrm{M}\right.$, type VI) reduction, was tested with a cell concentration of $2.5 \times 10^{6}$ in $2 \mathrm{ml}$ buffer. The patient's eosinophils, as well as his granulocyte fraction, failed to produce any detectable superoxide after PMA stimulation. Purified eosinophils from normals and patients with parasitosis at the same cell concentration and under the same conditions produce superoxide in amounts equivalent to or greater than the same number of unfractionated granulocytes.

\section{DISCUSSION}

Eosinophils from half of our CGD patients showed staining characteristic of NBT reduction after stimulation with PMA. This NBT reduction by CGD eosinophils was not expected since the enzymatic defect in neutrophils from CGD patients would be anticipated in other phagocytic cells. The NBT reduction in CGD eosinophils is probably not related to superoxide production as no superoxide could be detected by cytochrome $c$ reduction. However, unlike normal eosinophils, NBT reduction in the CGD eosinophils was confined to a small area of the cell near the nucleus and it is conceivable too little superoxide may have been produced by CGD eosinophils to be detectable by the cytochrome $c$ assay.

CGD is actually a group of disorders of intraleukocyte metabolism with similar clinical manifestations of recurrent infections due to different defects in the enzyme chain responsible for generating the respiratory burst when the cell is activated $(1,10)$. It may be classified into several hereditary patterns including Xlinked, autosomal recessive and possibly autosomal dominant (1). The CGD patients whose eosinophils reduced NBT included those with established X-linked and autosomal hereditary patterns. Thus, the staining, or lack of it, did not follow the same genetic pattern as the particular CGD defect. The eosinophil NBT reduction also could not be correlated with the presence or absence of cytochrome b or FAD (Table 1), a probable component of the NADPH oxidase system that is absent or deficient in some CGD patients (10). The FAD content was also examined and no correlation with eosinophil NBT reduction has been noted. Nathan et al. (11) reported that NBT reduction could be demonstrated within the phagocytic vacuoles of leukocytes from three brothers with CGD but only after a 30 - to 60 -min incubation (11). Whether this is an observation unique to one family or a generalized observation among CGD patients is not known.

Differential counts from peripheral blood smears revealed several of our CGD patients had a mild eosinophilia $(4.6 \pm 0.7 \%$ with a range of $3-10 \%$ ) and that eosinophils accounted for 7.1 $\pm 1.2 \%$ of the unfractionated granulocytes. Previous reports have found small numbers of NBT positive cells (up to $4 \%$ ) in CGD patients (2) which would be difficult to explain on a genetic basis. It is possible that these positive cells were actually stained eosinophils. It is also possible that a CGD patient with a marked eosinophilia might be confused with a heterozygote.

CGD neutrophils are able to perform other phagocytic functions after activation such as chemotaxis and phagocytosis despite their lack of oxygen radical production. The eosinophil NBT reduction was only noted after exposure to PMA indicating that some form of activation was necessary for the NBT reduction to occur in the CGD eosinophil. The absence of staining in the CGD neutrophils may indicate a basic difference in the metabolism of eosinophils and neutrophils not evident when testing normal granulocytes. The possible significance of eosinophil NBT reduction to host defense will require further studies.

\section{REFERENCES}

1. Gallin JI, Buescher ES, Seligmann BE, Nath J, Gaither T, Katz P 1983 Recent advances in chronic granulomatous disease. Ann Intern Med 99:657-674

2. Newburger PE, Cohen HJ, Rothchil SB, Hobbins JC, Malawista SE, Maloney MJ 1979 Prenatal diagnosis of chronic granulomatous disease. N Engl J Med 300:178-181

3. Roberts RL, Gallin JI 1985 Rapid method for isolation of normal human peripheral blood eosinophils on discontinuous Percoll gradients and comparison with neutrophils. Blood 65:433-440

4. Buescher ES, Gallin JI 1982 Leukocyte transfusions in chronic granulomatous disease. N Engl 3 Med 307:800-803

5. Seligmann BE, Fletcher MP, Gallin JI 1983 Histamine modulation of human neutrophil oxidative metabolism, locomotion, degranulation, and membrane potential changes. J Immunol 130:1902-1909

6. Ohno Y, Gallin JI 1985 Diffusion of extracellular hydrogen peroxide into intracellular compartments of human neutrophils. Studies utilizing the inactivation of myeloperoxidase by hydrogen peroxide and azide. $\mathrm{J}$ Biol Chem 260:8438-8446

7. Gabig TG, Lefker BA 1984 Deficient flavoprotein component of the NADPHdependent $\mathrm{O}_{2}{ }^{-}$generating oxidase in the neutrophils from three male patients with chronic granulomatous disease. J Clin Invest 73:701-705

8. Faeder EJ, Siegal LM 1973. A rapid micromethod for determination of PMN and FAD in mixtures. Anal Biochem 53:332-336.

9. Schliwa M, Pryzwansky KB, Entereuer Y 1982 Centrosome splitting in neutrophils: an unusual phenomenon related to cell activation and motility. Cell 31:705-717

10. Tauber Al, Borregaard N, Simons E, Wright J 1983 Chronic granulomatous disease: a syndrome of phagocyte oxidase deficiencies. Medicine 62:286-300

11. Nathan DG, Baehner RL, Weaver DK 1969 Failure of nitroblue tetrazolium reduction in the phagocytic vacuoles of leukocytes in chronic granulomatous disease. J Clin Invest 48:1895-1904 